\section{Biotransformation and toxicity of inhalational anaesthetics}

Michio Morio MD PhD, Osafumi Yuge MD PhD, Kohyu Fujii PhD
Anaesthetics are "xenobiotics," that is chemical compounds that are foreign to the human body. Volatile anaesthetics are distributed through the lung and blood to various organs. They can be metabolized by specific enzyme systems which have high activity in the liver but which are also present in the kidney and other organs.

The processes of distribution and elimination of drugs and their metabolites have been studied for each anaesthetic. ${ }^{1}$ Most anaesthetics are lipophilic which facilitates penetration of membrane barriers. The water/gas or blood/gas ratios and oil/gas or lipid/gas ratios of inhalational anaesthetics play an important role in their absorption and accumulation in the body. The volatile anaesthetic with the lowest solubility in the body is considered to be sevoflurane which has been recently placed on the market in Japan. Its water/gas or blood/gas ratio is similar to that of nitrous oxide, but the oil/gas or fat/gas ratio differs from that of nitrous oxide. It has, theoretically, a lower absorption and accumulation in the body.

Metabolism of a drug, in general, may lead to the formation of a metabolite that is much less toxic than the parent compound, but in some cases the toxicity is enhanced by metabolism.

Elimination of drug and its metabolites usually takes place in the urine, bile (faeces), and expired gas. This paper describes the current concept of biotransformation and toxicity of inhalational anaesthetics (Table I).

\section{Biotransformation}

The enzymatic reactions involved in hepatic drug metabolism can be classified into phase I and phase II reactions, that is, oxidation or reduction (phase I) and conjugation or synthesis (phase II) (Table II).

An important aspect is the properties of the enzymes that catalyze the metabolic conversions of drugs. Many of these enzymes are located in the microsome, that is in the membrane framents of the endoplasmic reticulum. In particular the enzymes of the oxidation system, such as cytochrome $\mathrm{P} 450$, are present in the microsome although some are found in the cytosol and mitochondria.

The membranes of rough and smooth endoplasmic reticulum, which are connected, are considered to contain the cytochrome P450 enzyme system. Singer's membrane model is well established and contains various kinds of enzymes. The membrane of the endoplasmic reticulum of liver cells resembles this schematic figure which shows the microsomal monooxidase enzyme system containing cytochrome P450 (Figure 1). Flavoproteins, $b_{5}$ and other enzymes concemed with electron transport are located in the membrane of endoplasmic reticulum near cytochrome P450.

The cytochrome P450 oxidation system is an electrontransport system containing terminal oxidase haemprotein and is associated primarily with membranes of the endoplasmic reticulum. Cytochromes, which are haemcontaining proteins, are termed P450, P448 or others depending on the wave length of the maximal absorption spectrum of the reduced form after carbon monoxide binding. However, in general, these enzymes are called cytochrome P450. It is known that P450 system contains enzymes which are induced by repeated administration of phenobarbitone and that the enzymes of the P448 system are induced by repeated administration of methylcholanthrane.

Figure 2 shows the differences of wave-length of the absorption spectrum of microsomes before and after repeated administration of phenobarbital or methylcholanthrane (3-MC). It is speculated that cytochrome P450 contains two drug-binding sites.

\section{Biotransformation of halothane (Figure 3)}

\section{Phase I reactions}

We investigated the oxidative (acrobic) ${ }^{2-5}$ and reductive (anaerobic) ${ }^{6}$ dehalogenation of halothane by the cytochrome P450 oxidation system. Two anaerobic volatile metabolites, chlorodifluoroethylene (CDE) and chlorotrifluoroethane (CTE) in the expired gas, ${ }^{7}$ and an aerobic metabolite of trifluoroacetic acid (TFA) and fluoride ion were found in the bile, saliva, blood and urine during and following administration of halothane. ${ }^{8-13}$

Also, the effects of enzyme-inducing drugs, such as phenobarbitone and other barbiturates which are used clinically, on aerobic and anaerobic dehalogenation of

Department of Ancsthesiology, Hiroshima University School of Medicine, Hiroshima, Japan 
TABLE I Solubility of anaesthetics

\begin{tabular}{llcccc}
\hline & $\begin{array}{l}\text { Water/gas } \\
\left(37^{\circ} \mathrm{C}\right)\end{array}$ & Bloodigas & Oillgas & Farlgas & MAC (\%) \\
\hline Sevoflurane & 0.36 & 0.59 & 53.7 & 47.7 & 1.71 \\
Isoflurane & 0.61 & 1.4 & 97.8 & 94.5 & 1.15 \\
Halothane & 0.7 & 2.3 & 224 & 185 & 0.75 \\
Enflurane & 0.8 & 1.9 & 98.5 & 105.0 & 1.68 \\
Methoxyflurane & 4.5 & 12.0 & 930 & 890 & 0.16 \\
Diethyl ether & 13.0 & 12.0 & 65 & 66 & 1.92 \\
& & & & & 1.22 \\
Nitrous oxide & 0.47 & 0.47 & 1.40 & 1.22 & 101 \\
\hline
\end{tabular}

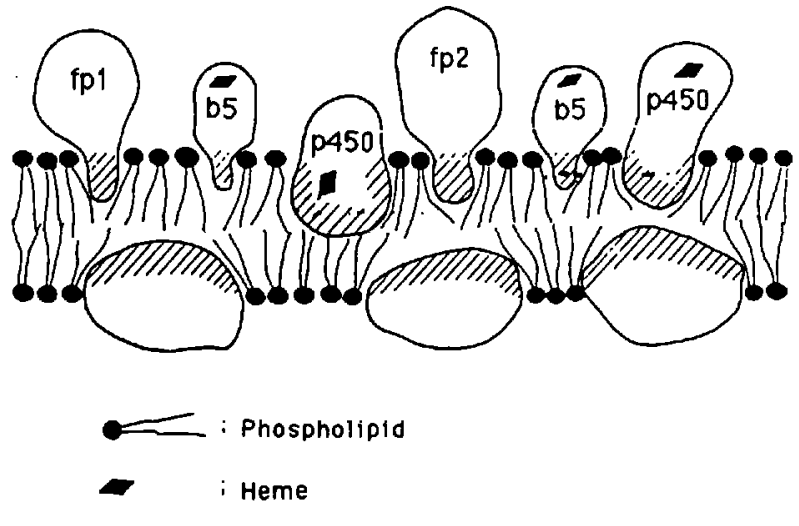

FIGURE 1 Microsomal enzyme model modified Singer's membrane model by Y. Yoshida. The shaded portion indicates hydrophobic. With permission from author and publisher.

TABLE II Enzymatic reactions

Pathways of drug metabolism

Phase I reactions

- Oxidation

- o-dealkylation

- alomatic hydroxylation

- aliphatic hydroxylation

- n-dealkylation

- sulfoxidation

- desulfuration

- epoxidation

- n-oxydation

- dehalogenation

- Reduction

- reductive dehalogenation

- others

Phase II reactions

- glucronic acid conjugation

- sulfate conjugation

- mercapturic acid formation

- amide syntesis

- other pathways of conjugation

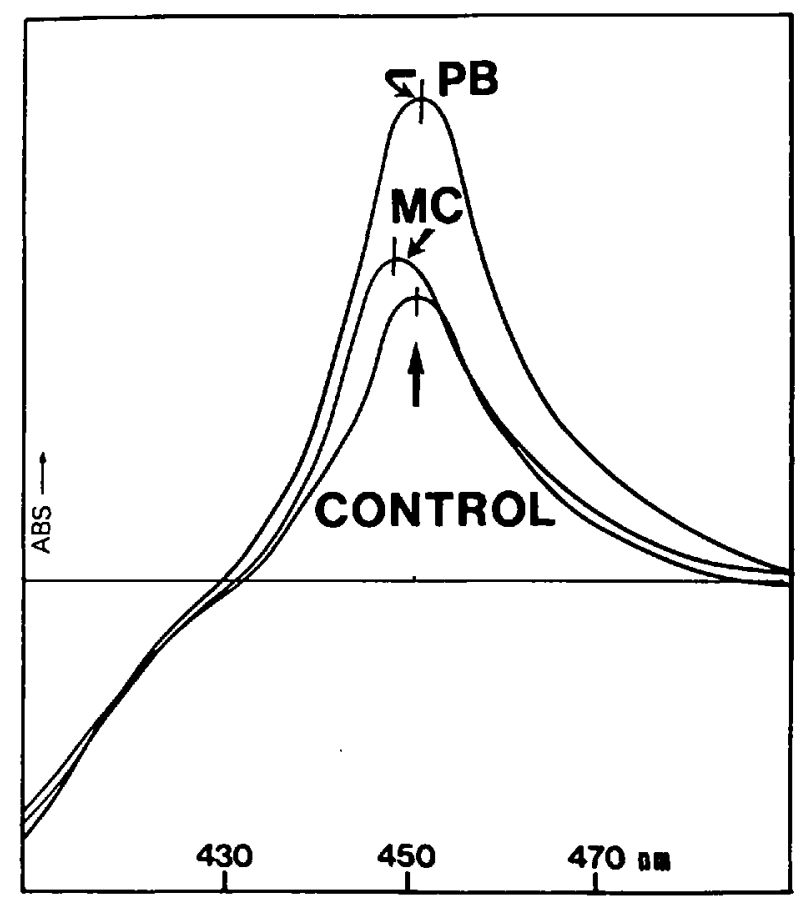

FIGURE 2 Wave-length of absorption spectrum of P450 and P448.

halothane were investigated. ${ }^{4}$ The greatest induction was seen after phenobarbitone and thiopentone and thiamylal produced significantly more induction than in the control or after pentobarbitone and secobarbitone.

\section{Phase II reactions}

The final step in the metabolism of a foreign compound involves conjugation with a water-soluble metabolite. The most common conjugation is glucuronic acid adduct catalyzed by UDP-glucuronyl transferase, which is localized in the endoplasmic reticulum. Cytosolic sulfotransferases catalyze the sulfation reaction of alcohol, bile acids, and a wide range of phenolic compounds. Glutathione, a cysteine containing tripeptide, is conjugated to 


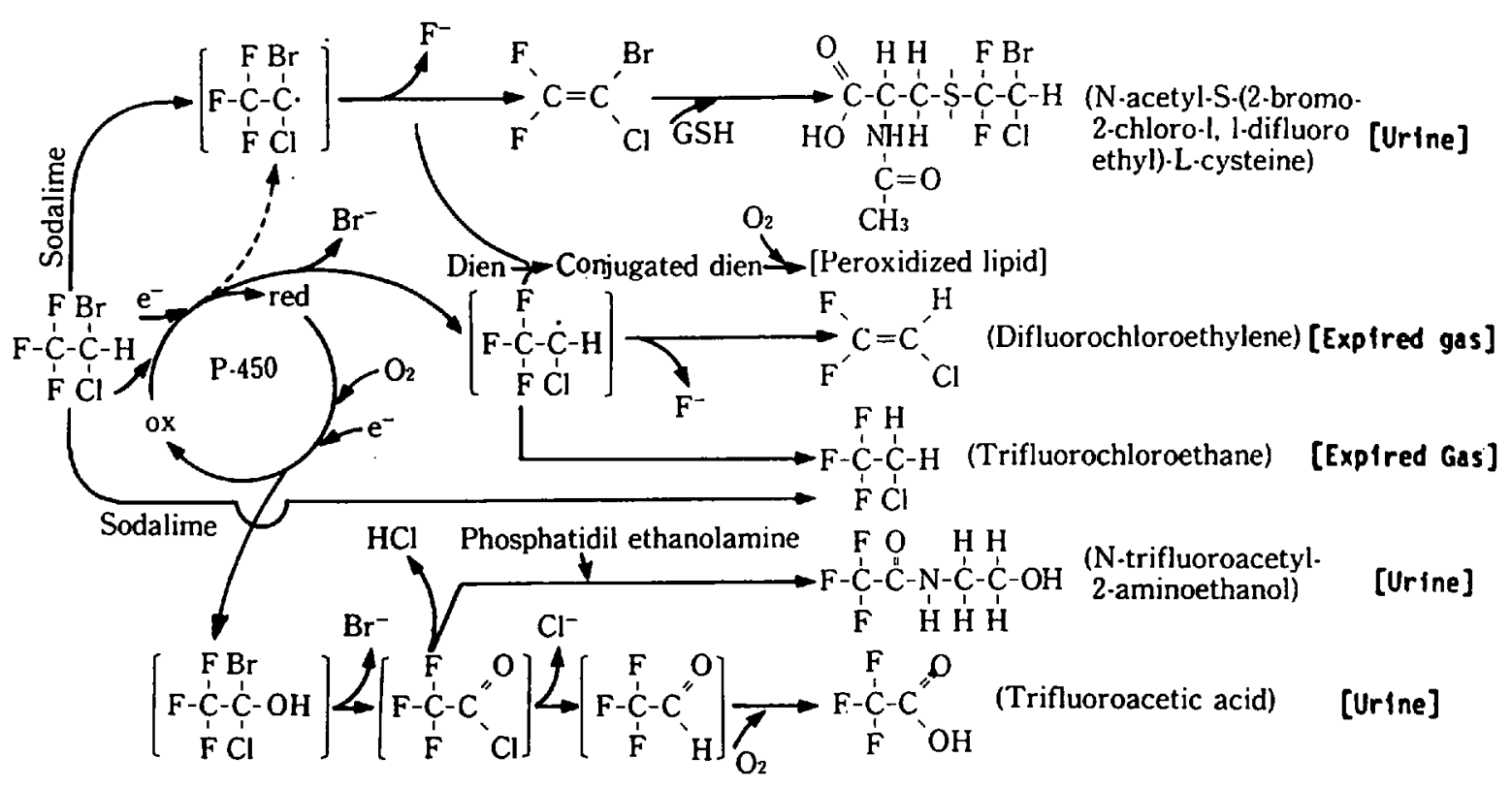

FIGURE 3 Biotransformation of halothanc.

xenobiotics by its free thiol group. This reaction is catalyzed by glutathione-s-transferase, but can occur, although at a slower rate, in its absence. Glutathione-stransferases ensure that these very reactive molecules are rapidly conjugated with glutathione, thus preventing toxic reactions. Under adverse conditions, the available glutathione substrate may be rapidly depleted so that potentially toxic compounds are accumulated.

\section{Factors affecting hepatic drug metabolism (enzyme induction)}

Clinical effects of enzyme induction only become apparent when the rate-controlling step in detoxication or elimination is affected. However, enzyme induction may have major effects in enhancing the metabolism of xenobiotics to toxic intermediates. The enzyme inducers reported in the literature are shown in the Table III. The rate and manner in which an individual metabolizes drugs are determined, in part, by genetic factors but these are rarely apparent unless they result in toxicity.

TABLE III Hepatic enzyme-inducing drugs

Phenobarbitone and other barbiturates

3-Methylcholanthrene

Dibutylhydroxytruene

3,4-Benzupyrene

Nicotinic acid amide

Nicotinic acid

Dichlorodiphenyltrichlorosthane

Polychlorinatedbiphenyl

Trihalometane

\section{Radical formation}

Repeated preanaesthetic administration of enzymeinducing drugs should be avoided. This is because we $\mathrm{e}^{15}$ and others have indicated that anaerobic metabolites were much increased in animals given repeated administration of phenobarbitone, and a great increase of free radical was detected during anaerobic metabolism of halothane.

\section{Enzyme inhibition}

In contrast to pre-anaesthetic enzyme induction, cimetidine inhibited anaerobic metabolism of halothane in an animal model.$^{16}$ Halothane inhibits the metabolism of phenytoin, warfarin, fentanyl and ketamine. Halothane anaesthesia inhibited the metabolism of enflurane in rats possibly by competing for the same drug-metabolizing sites on microsomal enzymes. Isoflurane administered simultaneously with or $30 \mathrm{~min}$ before halothane significantly inhibited oxidation metabolism of halothane. ${ }^{17}$

Our unpublished data also indicate that some drugs used clinically also inhibit anaerobic halothane metabolism in vitro. The simultaneous administration of thiamylal and thiopentone, known enzyme inducers, inhibited anaerobic metabolism of halothane. ${ }^{14}$

\section{Toxicity}

Drug metabolism has been considered to be detoxication. However, in recent years, some toxification by metabolism has been observed. Free radical formation $\left(\mathrm{CF}_{3} \mathrm{CHCl}\right)$, by reconstituted cytochrome $\mathbf{P} 450$ enzyme system, has been found under anaerobic conditions with halothane. ${ }^{4.5}$ 
Hypoxia plays an important role in hepatic disorders of animals and anaerobic halothane metabolism is an important role in intermediate radical formation. Anaerobic halothane metabolism and free radical formation were found to be increased by hypoxia.

\section{Possible mechanisms of hepatotoxicity of anaesthetics} There are at least two possible mechanisms for halothane hepatotoxicity: in the direct toxic reaction related to the reductive pathway, and the immune mediated reaction in which the antigen is associated with the oxidative and/or reductive route. Hypoxia, obesity, genetic factors, immunological factors, preanaesthetic conditions such as hypoproteinaemia, depressed serum glutathione level and drug interactions might modify the biotransformation of anaesthetics and may cause an associated factor of hepatotoxicity.

\section{Serum fluoride concentration}

The elevated serum fluoride concentration after methoxyflurane is considered to be a cause of nephrotoxicity. Defluorination ofmethoxyflurane is also related to cytochrome P450 enzyme system. Serum fluoride concentrations are increased after methoxyflurane anaesthesia and many reach nephrotoxic levels of $50 \mu \mathrm{m} \cdot \mathrm{L}^{-1}$. Mean serum fluoride levels achieved after other anaesthetics is less than $50 \mu \mathrm{m} \cdot \mathrm{L}^{-1}$. However, we cannot say that every patient is safe, because some may have higher enzymatic activities.

\section{Degradation of anaesthetics by soda lime}

Degradation of anaesthetics by soda lime is not biotransformation, but all volatile anaesthetics are decomposed by soda lime before inhalation. ${ }^{18.19}$ Some products of degradation are highly toxic, but, fortunately they are produced in very small quantities and are usually diluted by a semiclosed anaesthetic circuit. The $\mathrm{CO}_{2}$ absorber and/or inhalational methods should be improved to prevent degradation of anaesthetics.

\section{Summary}

In summary, anaesthetics and drugs used perioperatively are all xenobiotics and can be metabolized mainly by microsomal enzyme systems, which have a high activity in the liver. These enzyme systems are induced by repeated pre-administration of drugs, such as barbiturates and others which are used during the preoperative period. However, according to some reports, aerobic and anaerobic metabolism is inhibited by the simultaneous administration of drugs, such as isoflurane and halothane, halothane and enflurane, and cimetidine and halothane.

Hypoxia is also an important factor in hepatic disorders and it is well known that anaerobic metabolism of halothane is increased by hypoxia and its intermediate production produces a free radical. Theoretically, this free radical is involved in hepatic disorders.

In practice, in order to prevent hepatic dysfunction before, during and after anaesthesia, hypoxia and repeated pre-administration of enzyme-inducing drugs should be avoided. However, the choice and combination of drugs which inhibit drug metabolism and prevent hepato and/or nephro toxicity should be examined by further investigation.

\section{References}

1 Cohen EN, Van Dyke RA. Metabolism of Volatile Anesthetics. Massachusetts: Addison-Wesley, 1977.

2 Fujii K, Morio M, Kikuchi $H$. A possible role of cytochrome $\mathrm{P}-450$ in anaerobic dehalogenation of halothane. Biochem Biophys Res Commun 1981; 101: 1158-63.

3 Fujii $K$, Miki N, Sugiyama T, Morio M, Yamano $T$, Miyake $Y$. Anaerobic dehalogenation of halothane by reconstituted liver microsomal cytochrome P450 enzyme system. Biochem Biophys Res Commun 1981; 102: 507-12.

4 Fujii $K$, Miki $N$, Kanashiro $M$ et al. A spin trap study on anaerobic dehalogenation of halothane by a reconstituted liver cytochrome P-450 enzyme system. J Biochem 1982; 91 : 415-8

5 Fujii $K$, Miki N, Sugiyama T, Morio M, Yamano $T$, Miyake $Y$. Anaerobic dehalogenation of halothane by a reconstituted mixed function oxidase system of rabbit liver microsomes. In: Ryo Sato, Ryuichi Kato (Eds.).

Microsomes, Drug Oxidation and Drug Toxicity. Tokyo, Japan Scientific Societies Press. 1982; 511-2.

6 Nakao $M$. Personal communication

7 Mukai S, Morio M, Fujii K, Hanaki C. Volatile metabolites of halothane in the rabbit. Anesthesiology 1977; 47: $248-51$.

8 Morio M, Fujii K, Takiyama R, Chikasue F, Kikuchi $H$ Ribaric $L$. Quantitative analysis of trifluoroacetate in the urine and blood by isotachophoresis. Anesthesiology 1980; 53: 46-9.

9 Takiyama $R$, Morio $M$, Fujii $K$ et al. Clinical effects of halothane concentration on trifluoroacetic acid excretion in urine. Hiroshima J Med Sci 1985; 34: 377-80.

10 Okida $M$, Kikuchi $H$, Fujii $K$. Concentration dependence of halothane metabolism in rabbits. Hiroshima $J$ Mcd Sci 1986; 35: 15-20.

11 Mirkov MI, Morio M, Kawahara M, Kinoshita H. Fujii K. Excretion of trifluoroacetic acid as a mctabolitc of halothane in digestive juices. Journal of Anesthesia 1988; 2: $161-4$

12 Kawahara M, Morio M, Akita S, Takesita $H, F u j i i$ $K$. Salivary excretion of trifluoroacetic acid (TFA) after halothane anesthesia. Journal of Anesthesia 1988; 2: 133-8. 
13 Kawaguchi $R$, Fujii $K$, Yuge $O$, Hossain D. Biliary excretion of TFA, a metabolite of halothane, in the rabbit determined by ion-chromatography. Hiroshima $\mathbf{J}$ Med Sci 1989; 38: 27-34.

14 Taira $Y$. Personal communication.

15 Fujii K, Morio M, Kikuchi H, Ishihara S, Okida M, Ficor $F$. In vivo spin-trap study on anaerobic dehalogenation of halothane. Life Sci. 1984; 35: 463-8.

16 Plummer $J L$, Wanwimolruk $S$, Jenner MA, Hall $P$ de la $M$, Cousins $M J$. Effects of cimetidine and ranitidine on halothane metabolism and hepatotoxicity in an animal model. Drug Metab Dispos 1984; 12: 106-10.

17 Fiserova-Bergerova V, Dolan DF. Transient inhibitry effect of isoflurane upon oxidative halothane metabolism. Anesth Analg 1985; 64: 1171-7.

18 Hanaki C, Fujii K, Morio M, Tashima T. Decomposition of sevoflurane. Hiroshima J Med Sci 1987; 36: 61-7.

19 Takeyoshi S. Stability of sevoflurane in sodalime. Hiroshima J Anesth 1990 (in press).

20 Alvares AP, Schilling $G$, Levin $W$, Kuntzman $R$. Studies on the induction of CO-binding pigments in liver microsomes by phenobarbital and 3-methylcholanthrene. Biochem Biophys Res Commun 1967; 29: 521-6. 
Michio Morio, MD PhD, Osafumi Yuge MD PhD, Kohyu Fujii PhD

\section{Biotransformation et toxicité des anesthé- siques d'inhalation}

Les anesthésiques généraux sont xénobiotiques, c'est-àdire sont des composés étrangers au corps humain. Les agents volatils sont distribués par le poumon et le sang à divers organes. Ils peuvent être métabolisés par des systèmes enzymatiques spécifiques qui ont une activité élevée dans le foie mais qui sont aussi présents dans le rein et d'autres organes.

Chaque agent anesthésique a fait l'objet d'études de distribution, d'élimination ainsi que d'élimination des métabolite.' La plupart des agents anesthésiques sont liposolubles ce qui facilite la pénétration des barrières membranaires. Le coefficient eau/gaz, sang/gaz et le rapport huile/gaz ou lipides/gaz des agents anesthésiques d'inhalation joue un rôle important dans leur absorption et leur accumulation dans l'organisme. Le volatil avec la plus faible solubilité dans le corps est considéré être le sevoflurane, qui a récemment été mis sur le marché au Japon. Son coefficient eau/gaz ou sang/gaz est semblable à celui du protoxyde d'azote, mais le coefficient huile/gaz ou lipide/gaz diffère de celui du protoxyde d'azote. Il présente en théorie une absorption plus faible et moins d'accumulation dans l'organisme.

Le biotransformation d'une substance, en général, peut conduire à formation d'un métabolite qui est beaucoup moins toxique que le composé d'origine, et dans quelques cas cette toxicité est augmentée par le métabolisme.

L'élimination des médicaments et de leur métabolite se fait habituellement par l'urine, la bile et dans les gaz expirés. Ce travail décrit les concepts actuels de biotransformation et de toxicité des anesthésiques d'inhalation. (Tableau I, voir page Scxvii).

\section{Biotransformation}

Les réactions enzymatiques impliquées dans le métabolisme hépatique peuvent être classées en réaction de phase l et de phase II, c'est-à-dire oxydation ou réduction (phase I) et conjugaison ou synthèse (phase II) (Tableau II, voir page Scxvii).

Les enzymes ont la propriété importante de catalyser la biotransformation des médicaments. Plusieurs de ces enzymes sont localisés dans les microsomes, c'est-à-dire dans les fragments membranaires du réticulum endoplasmique. En particulier, les enzymes du système d'oxydation, comme le cytochrome $\mathrm{P} 450$ sont présents dans les microsomes alors que d'autres se retrouvent dans le cytosol et les mitochondries.

Les membranes du réticulum endoplasmiques sont considérées comme les sites du système cytochrome $\mathrm{P} 450$. Le modèle membranaire de Singer est bien établi et contient de nombreuses variétés d'enzymes. La membrane du réticulum endoplasmique de l'hépatocyte possède à peu près cette forme schématique (figure 1 , voir page Scxvii) qui montre dans le microsome le système d'enzyme mono-oxydase contenant le cytochrome P450. Les flavoprotéines, la $b_{5}$ et les autres enzymes impliqués dans le transport des électrons sont situées dans la membrane du réticulum endoplasmique près du cytochrome $\mathrm{P} 450$.

Le système d'oxydation cytochrome P450 est un système à transport d'électrons qui contient l'oxydase terminale hémoprotéique et est associé d'abord aux membranes du réticulum endoplasmique. Les cytochromes, qui sont des protéines contenant un groupe hème, sont nommées $\mathrm{P} 450, \mathrm{P} 448$ ou autres noms dépendant de la longuer d'onde du spectre d'absorption maximal de la forme réduite après liaison au monoxyde de carbone. Cependant, en général ces enzymes sont appelées cytochrome P450. Il est bien connu que le système P450 contient des enzymes qui sont induits par l'administration répétée de phénobarbital et que les enzymes du système P448 sont induits par l'administration répétée de méthylcholanthrène.

La figure 2 (voir page Scxvii) montre les différences de longueurs d'onde du specte d'absorption des microsomes avant et après l'administration répétée de phénobarbital ou de méthylcholantrène. On spécule que le cytochrome P450 contient deux sites de liaisons aux médicaments.

\section{Biotransformation de l'halothane (figure 3, page Scxviii)}

\section{Réaction de phase I}

Nous avons évalué la déhalogénation oxydative (aérobique) $^{2-5}$ et réductrice (anaérobique) ${ }^{6}$ de l'halothane par le système d'oxydation cytochrome $\mathrm{P} 450$. Nous avons trouvé deux métabolites anaérobiques du volatil, le chlorodifluoroéthylène (CDE) et chlorotrifluoroéthane (CTE) dans les gaz expirés, ${ }^{7}$ un métabolite aérobique de l'acide trifluoroacétique (TFA) ainsi que des ions fluor 
dans la bile, la salive, le sang et l'urine pendant et après l'administration d'halothane. ${ }^{8-13}$

De plus, les effets des agents d'induction enzymatique tels que le phénobarbital et autres barbituriques utilisés en clinique, sur la déhalogénation aérobique et anaérobique de l'halothane, ont aussi fait l'objet de recherche. ${ }^{4}$ La plus forte induction s'est produite après phénobarbnital ; le thiopental, le thiamylal ont produit plus d'induction que les contrôles ou après pentobarbital ou secobarbital.

\section{Réactions de phase II}

L'étape finale dans la biotransformation d'un composé xénobiotique implique la conjugaison avec un métabolite hydrosoluble. La conjugaison la plus fréquente se fait avec l'acide glucoronique ; elle est catalysée par l'UDPglucuronyl transférase, qui est aussi localisé dans le réticulum endoplasmique. Les sulfotransférases cytosoliques catalysent les réactions de sulfatation de l'alcool, des acides biliaires, et d'un vaste éventail de composés phénoliques. Le glutathion, un tripeptide qui contient de la cystéine, est conjugé aux molécules étrangères par son groupe thiol libre. Cette réaction est catalysée par la glutathion-s-transférase, mais peut se produire avec une vitesse plus lente en son absence. La glutathion-stransférase fait en sorte que ces molécules très réactives sont rapidement conjugées avec le glutathion, prévenant ainsi les réactions toxiques. Sous des conditions défavorables, le substrat d-glutathion disponible peut être rapidement diminué de telle sorte que certains composés potentiellement toxiques s'accumulent.

\section{Les facteurs qui influencent le métabolisme hépatique des médicaments (induction enzymatique)}

Les effets cliniques de l'induction enzymatique deviennent apparents seulement quand la cinétique de détoxification ou de l'élimination est touchée. Cependant, l'induction enzymatique peut avoir des effets majeurs en augmentant le métabolisme des substances xénobiotiques en intermédiaires toxiques. Les inducteurs enzymatiques mentionnés dans la littérature sont présentés dans la Tableau III (voir page Scxviii). La vitesse et la manière par lesquelles un individu métabolise les substances pharmaceutiques sont déterminées en partie par des facteurs génétiques mais ceux-ci sont rarement apparents à moins qu'il y ait démonstration de toxicité.

\section{Formation de radicaux}

L'administration répétée de substances inductrices devrait être évitée dans la période pré-anesthésique. Ceci parce que nous ${ }^{15}$ et d'autres ont montré des métabolites anaérobiques présents en plus grande quantité chez les animaux qui avaient reçu des administrations répétées de phénobarbital et l'on a mis en évidence une augmentation importante de radicaux libres pendant le métabolisme aérobique de l'halothane.

\section{Inhibition enzymatique}

$\mathrm{Au}$ contraire de l'induction enzymatique préanesthésique, la cimétidine inhibe le métabolisme anaérobique de l'halothane dans un modèle animal. ${ }^{16} \mathrm{~L}$ 'halothane inhibe le métabolisme du phénytoin, de la warfarine, du fentanyl et de la kétamine. L'anesthésie à l'halothane a inhibé le métabolisme de l'enflurane chez des rats possiblement par compétition pour le même site de métabolisme sur les enzymes du microsome. L'isoflurane administré simultanément avec, ou 30 minutes avant l'halothane diminue de façon significative le métabolisme oxydatif de l'halothane. ${ }^{17}$

Nos données non publiées indiquent aussi que certaines substances utilisées en clinique vont aussi inhiber le métabolisme anaérobique de l'halothane in vitro. L'administration simultanée de thiamylal et de thiopental, inducteurs enzymatiques connus, va inhiber le métabolisme anaérobique de l'halothane. ${ }^{14}$

\section{Toxicité}

On a considéré le métabolisme des médicaments comme étant de la détoxification. Cependant, dans les dernières années, des processus métaboliques ont produits de la toxification. On a mis en évidence sous condition anaérobique avec l'halothane, la formation de radicaux libres $\left(\mathrm{CF}_{3} \mathrm{CHCl}\right)$, par un système enzymatique cytochrome $\mathrm{P} 450$ reconstitué. ${ }^{4,5}$

L'hypoxie joue un rôle important dans les désordres hépatiques chez les animaux et le métabolisme anaérobique de l'halothane joue un rôle important dans la formation des radicaux intermédiaires. Le métabolisme anaérobique de l'halothane et la formation de radicaux libres sont augmenté par l'hypoxie.

\section{Mécanisme possible d'hépatotoxicité des anesthésiques} Il y a au moins deux mécanismes possibles pour expliquer l'hépatotoxicité à l'halothane ; une réaction directe et toxique reliée à la voie réductrice, et une réaction immunitaire dans laquelle l'oxygène est associé, par la voie oxydative et/ou réductrice. Des facteurs tels l'hypoxie, l'obésité, certains facteurs génétiques, des facteurs immunologiques, des conditions pré-anesthésiques telles que l'hypoprotéinémie, une diminution du niveau sérique de glutathion, et certaines interactions médicamenteuses peuvent modifier la biotransformation des agents anesthésiques et ainsi devenir des facteurs associés à une hépatotoxicité.

\section{Concentration en ions fluor}

La concentration élevée de fluor après l'anesthésie au 
méthoxyflurane constitue une cause de néphrotoxicité. La défluoration du méthoxyflurane est aussi en relation avec le système enzymatique cytochrome P450. Les concentrations de fluor sérique augmentent après l'anesthésie au méthoxyflurane et peuvent atteindre des niveaux néphrotoxiques à $50 \mu \mathrm{m} \cdot \mathrm{L}^{-1}$. Des niveaux inférieurs de fluor sérique sont obtenus après l'utilisation d'autres agents anesthésiques. Cependant, on ne peut assurer que chaque patient sera en sécurité lorsque certains peuvent avoir des niveaux d'activité enzymatiques plus élevés.

Dégradation des agents volatils par la chaux sodée La dégradation des volatils par la chaux sodée n'est pas de la biotransformation, mais tous les volatils sont décomposés par la chaux avant l'inhalation. ${ }^{18.19}$ Quelques produits de dégradation sont hautement toxiques mais sont heureusement produits en très petites quantités et sont aussi habituellement dilués par le circuit anesthésique semi-fermé. L'absorbeur de $\mathrm{CO}_{2}$ et/ou les méthodes d'inhalation devraient être améliorés pour prévenir la dégradation des anesthésiques.

\section{Résumé}

Les agents anesthésiques et autres médicaments utilisés dans la période péri-opératoire sont tous xénobiotiques et peuvent être métabolisés surtout par les systèmes enzymatiques du microsome, qui ont une activité élevée dans le foie. Ces systèmes enzymatiques sont induits par une pré-administration répétée de certaines substances, telles les barbituriques et autres qui sont utilisés pendant la période pré-opératoire. Cependant, selon certains travaux, le métabolisme aérobique et anaérobique est inhibé par l'administration simultanée de substances, telles I'isoflurane et l'halothane, l'halothane et l'enflurane, et la cimetidine et l'halothane.

L'hypoxie est aussi un facteur important dans l'apparition des problèmes hépatiques et il est bien connu que le métabolisme anaérobique de l'halothane est augmenté par l'hypoxie, et à un stage intermédiaire produit des radicaux libres. Sur une base théorique ces radicaux libres sont impliqués dans les dysfonctions hépatiques.

En pratique, dans le but de prévenir la dysfonction hépatique avant, pendant et après l'anesthésie, il faut éviter l'hypoxie et l'administration répétée d'inducteurs enzymatiques avant l'anesthésie. Cependant, le choix et la combinaison de substances qui inhibent le métabolisme des médicaments et préviendront l'hépato ou la néphrotoxicité devrait faire l'objet de travaux dans le futur.

\section{Références \\ (Voir page Scxix)}

